\title{
Average Distance Estimation in Randomly Deployed Wireless Sensor Networks (WSNs): An Analytical Study
}

\section{Cüneyt Sevgi*}

Department of Computer Technologies and Information Systems,

Bilkent University,

Ankara, Turkey

E-mail: csevgi@bilkent.edu.tr

*Corresponding author

\begin{abstract}
A Wireless Sensor Network (WSN) is an energy-scarce network in which the energy is primarily dissipated by the sensor nodes during data transmission to the base station (BS). The location of the BS in a WSN dramatically affects the energy dissipation, the throughput, and the lifetime. While in a number of studies the optimal positioning of a BS is considered, the system parameters are optimized when the BS location is known in advance in many others. In this paper, we provide a general-purpose mathematical framework to find the expected distance value between every point within any n-sided simple polygon shaped sensing field and an arbitrarily located BS. Having the knowledge of this value is very imperative particularly in random deployment as it is used for energy-efficient clustering. Although similar derivations appear in the related literature, to the best of our knowledge, this study departs from them, since our derivations do not depend on the shape of the sensing field and the orientation of BS relative to it.
\end{abstract}

Keywords: WSNs; wireless sensor networks; average distance; estimation; random deployment; base station.

Reference to this paper should be made as follows: Author(s) (2018) 'paper title ', Int. J. Sensor Networks, Vol. X, No. Y4, pp.000-000.

Biographical notes: Cüneyt Sevgi received his Ph.D. and M.Sc. degrees from Informatics Institute of Middle East Technical University (METU), Ankara, Turkey, both in Information Systems, in 2003 and 2009, respectively. He received his B.Sc. degree in Department of Physics from METU in 1998. He has been working for Department of Computer Technologies and Information Systems, Bilkent University, Ankara, Turkey. His research interests include Software Engineering, Software Management, Data Communications and Networking, Wireless Communication, Wireless Sensor Networks, and the Internet Traffic.

\section{Introduction}

A wireless sensor network (WSN) is a network which is formed by numerous sensor nodes deployed over a sensing field for a wide range of monitoring applications. These nodes are usually battery-powered and they communicate with each other through a wireless channel in an ad hoc fashion without a need for a fixed backbone infrastructure. In these networks, power is the main scarce resource due to the size limitations of nodes. WSNs also suffer from the scarcity of other resources such as limited storage and bandwidth, application-specific sensing capabilities, and moderate onboard processing power. Although there are many constraints, limitations and challenges during the operation of WSNs, numerous applications, which had not been conceived before the launch of WSNs, can be easily realized.

Each sensor node in a WSN is typically responsible for monitoring a physical phenomenon (i.e., coverage) around its vicinity. These sensing devices then work collaboratively to route (i.e., connectivity) the sensed data to the base station (BS). Further, when coverage and connectivity are tackled jointly (i.e., connected coverage), they are considered as two primary performance metrics in a given deployment scenario as is stated in Maher et al. (2016).

The sensor nodes are positioned according to either deterministic or random deployment. In the deterministic scenario, the locations of the sensor nodes are known in advance. On the contrary, in random deployment, the locations of the sensor nodes are not deterministic as the term itself also implies. It has been observed that randomly deployed WSNs (RDWSNs) are used more often than their deterministic counterparts because RDWSNs have a higher potential to be devised in real-life scenarios, especially when there is a need to monitor a phenomenon taking place in hostile and inaccessible environments. 
In a deterministic deployment scenario, as there is prior knowledge about the locations of the deployed nodes, the average distance between each node and its neighbours, and similarly the average distance between deployed nodes and the $B S$, can easily be calculated. However, in the random deployment scenarios, the above mentioned distances, which indeed affect the energy consumption and thus the lifetime of an application, are not known before the deployment. Therefore, a probabilistic approach is required to estimate this average distance which would provide a beneficial tool for the network designers. For example, for a given number of sensor nodes that fulfil the required system parameters such as connected coverage, the network designer might need to estimate, if possible, the average distance between each sensor and a BS to adopt either multi-hop communication or single-hop communication before the deployment.

In a number of RDWSN applications, each sensor node is assumed to reach the BS within a single-hop. However, in these applications which exploit direct communication, it is observed that a set of sensor nodes, which is distant from the BS, consumes a considerable amount of energy because it needs to perform long-haul direct transmissions. Therefore, these nodes, which are distant from the BS, tend to die early and thus shorten the lifetime of the network. To tackle this problem, multi-hop communication is usually taken into consideration because it is more energy-efficient than direct communication in such dense environments. In multi-hop WSNs, however, the nodes close to the BS have to forward data for the rest of the nodes that are away from the BS. Thus, they are again more likely to die earlier, causing the network to become partitioned and the network lifetime to get shortened. This is known as the energy-hole problem as is defined in Gong et al. (2013) and Du et al. (2015). Even before attempting to tackle an energyhole problem, the use of this estimated average distance to BS is imperative in some cases. For example, consider a WSN in which $N$ nodes are deployed randomly. Let us assume that the average distance between these nodes and the BS is estimated and it is denoted by $\bar{d}$. And further assume that $d_{0}$ is the maximum transmission range (MTR) of the sensor nodes. The MTR is a threshold value related to the physical attributes of the nodes such as initial battery power, wireless circuitry, etc. as is stated in Du et al. (2015). If $\bar{d}$ is found to be greater than $d_{0}$, the use of single-hop communication in this network is not viable because we already know that some nodes would not reach the BS. In this special case, there is no other way than using multi-hop communication. For such a justification, a network designer requires the knowledge of this average distance, $\bar{d}$, to compare it with the $d_{0}$ value.

In addition, the energy-hole problem is inevitable in most of the random deployment scenarios. Yet, the effects of this problem can be reduced by adopting clustering. Clustering is used as the most common technique due to its direct impact on energy-efficiency, network scalability and, more importantly, on the overall network lifetime. This is the reason why there are numerous studies on this subject in the related literature. The reader is encouraged to refer to recent and comprehensive surveys of Tyagi and Kumar (2013), Afsar and Tayarani-Najaran (2014), and Soroush et al. (2012) for an overview of different clustering schemes. In a clustered
WSN, the sensor nodes are basically grouped into clusters based on the proximity of the neighbouring nodes, the average distance to the $B S$, energy levels, etc. The problem of finding the average distance between the sensor nodes and a BS is very important not only for comparing the average value with the MTR value as is mentioned before but also for using this average value during the selection process of clustering schemes.

With the above problems in mind, this paper puts forward a general-purpose mathematical framework to find the expected distance value between every point within any $n$-sided simple polygon shaped sensing field and an arbitrarily located BS whose position is known a priori. The contribution of this paper lies in estimating the average distance between a given number of sensor nodes deployed randomly and uniformly over a sensing field and a BS which would provide a beneficial tool for the network designers. The formulations in our framework are important because these might provide a network designer with the opportunity to estimate the system parameters before the deployment. Having the knowledge of this expected distance value is very imperative particularly in random deployment as it is used typically to evaluate the energy-efficient cluster size either to improve the lifetime or to compare it with other counterpart cluster-based schemes.

The rest of this paper is organised as follows: Section 2 introduces a review of related literature devoted to this field to identify the reasons of clustering and the importance of optimum number of clusters in RDWSNs. Section 3 introduces the network model employed and the relevant assumptions used throughout the paper. Section 4 illustrates our derivations of $E\left[d_{t o B S}\right]$ and $\bar{d}$ for a number of deployment scenarios used in potential real-life WSN applications. The last section concludes our study.

\section{Related Work}

A WSN generally contains numerous battery-operated sensor nodes that have limited resources such as energy, bandwidth, memory, and processing power (Qingguo and P. (2017)). The most common goal of employing WSNs is to reduce energy consumption as much as possible while fulfilling a given set of system requirements for continuous and effective connected coverage. To achieve this objective, there are three major issues that should be considered by a network designer: 1) the data routing mechanism 2) the location of the BS, and 3 ) the deployment of the nodes or the coverage of the sensing field as mentioned in Guney et al. (2012). These three issues are indeed highly interrelated, which virtually cause the design of a WSN to be challenging.

In a WSN, the sensor nodes typically route the sensed data wirelessly to a single BS or multiple BSs. Moreover, a substantial amount of energy by the sensor nodes is consumed during the wireless transmission of the sensed data as is reported in Kulkarni and Venayagamoorthy (2011) and Alia (2017). Due to the fact that these nodes are severely power-constrained and the wireless communication is mainly dependent on the distance, the location of $\mathrm{BS}(\mathrm{s})$ and the physical distance(s) from the nodes are significant. For the transmission of these sensed data, the sensor nodes use either single-hop or alternatively multi-hop communication until data reaches the BS. The selection of single-hop or multi-hop 
routing of the sensed data depends on many issues: the node density, the deployment method, the nodes' initial energy, MTR, etc., as well as the positioning of the BS(s). In addition to its importance in data routing, the location of the BS relative to sensor nodes is also one of the key determinants of the network lifetime. For this reason, there are a lot of studies that have been carried out for analysing the effects of positioning and other attributes of $\mathrm{BS}(\mathrm{s})$ in the related literature.

There are two main sets of problems studied by several works that focus on the location of a BS in WSNs. In the first one, the researchers find an optimal transmission scheme for a given BS location. In the second one, they find an optimal BS location for a given set of constraints as is cited in Akkaya et al. (2007).

As is mentioned earlier in this study, the most common objective of devising WSNs is to reduce energy consumption as much as possible while fulfilling a given set of system requirements for continuous and effective connected coverage. To achieve an accepted level of connected coverage while conserving energy, the topology management techniques in WSNs are exploited (Younis et al. (2014)). Topology management in WSNs can be done through deterministic node placement or performed autonomously after random deployment by human intervention to a certain extent. Authors in Younis et al. (2014) categorize five techniques and algorithms used in topology management: Node Discovery, Sleep Cycle Management, Power Control, Movement Control and Clustering.

Clustering has been widely used in WSNs to achieve scalability and energy efficiency, where nodes of a WSN may be grouped to form a hierarchical topology as is stated in Younis et al. (2014). Moreover, other than stabilizing network topology, one clustering mechanism or another is used for the following objectives: increasing scalability and/or fault tolerance, taking advantage of data aggregation/fusion and/or load balancing, and extending network lifetime Afsar and Tayarani-Najaran (2014).

LEACH (Low-Energy Adaptive Clustering Hierarchy) presented in Heinzelman et al. (2002) is a pioneering paper that proposes a cluster-based solution to prolong lifetime; therefore, it is unquestionably the most widely-used and influential work. Most of the cluster-based WSN architectures have been inspired by LEACH and they either ignore LEACH's inherent weaknesses and disregard its strong assumptions or simply adapt LEACH to a different scenario. In fact, even a presence of a survey of Tyagi and Kumar (2013) that solely reviews a number of variants of LEACH itself is an indicator of the considerable impact of LEACH in the WSN domain.

LEACH mainly integrates the concept of energyefficient cluster-based routing into medium access to prolong the system lifetime in a single-hop communication mode. It addresses energy consumption minimization problems by making use of a distributed round-based algorithm. In each round of this distributed algorithm, it is expected that there is initially a chosen number of clusters. After the completion of each round, clusterheads can be re-elected periodically and randomly to balance the energy consumption. Thus, LEACH highly relies on the optimal number of clusters $\left(k_{\text {opt }}\right)$ and in each round it is assumed that WSN consists of $k_{o p t}$ number of clusters. During LEACH's setup phase, each sensor node tries to become a cluster head according to the probability model. Further, the authors discuss the trade-off between the inter-cluster communication and the intra-cluster communication that balances energy consumption, and they derive analytical expressions from simplifying approximations. They also present analytical and simulation results and demonstrate the high performance of LEACH when compared with minimum transmission energy (MTE) and static clustering.

Regardless of the clustering technique employed or the communication mode (i.e., multi-hop or single-hop) exploited, a RDWSN application can take advantage of clustering if and only if the application is grouped into $k_{o p t}$ clusters. While grouping the nodes into clusters different from $k_{\text {opt }}$, the energy consumption of the RDWSN may become inefficient and the application's lifespan may shorten even faster. The authors in Amini et al. (2011) and Amini et al. (2012) state that $k_{o p t}$ is a function of the expected distance value (i.e. $E\left[d_{t o B S}^{n}\right]$ ) between the sensor nodes and the BS. They provide a complete theoretical framework for the characterization of cluster size that minimizes the total energy expenditure in such networks where all sensors communicate data through their elected clusterheads to the BS in a decentralized fashion. The analytical outcomes are given in terms of closed-form expressions for various widelyused network configurations. Extensive simulations are performed for the validation purposes when three clusterbased architectures, namely LEACH, LEACH-Coverage Tsai (2007), and DBS Amini et al. (2011) are used. There can be several scenarios where the BS is positioned relative to the sensing field. A majority of these scenarios have been studied by Amini et al. (2012) and $E\left[d_{\text {toBS }}^{n}\right]$ and thus $k_{\text {opt }}$ values are derived for $n=1, n=2$, and $n=4$ when the sensing field is a disc or a square. Authors address the problem of determining the $k_{\text {opt }}$ of randomly deployed nodes when the BS is located inside the field, on the perimeter and outside (on the axis of) the sensing field. They also analyse the $E\left[d_{t o B S}\right]$ formulations by varying the locations of the BS as follows: The BS is located 1) in the center 2) on the perimeter 3 ) outside the sensing field (on the axis of symmetry). Although the formulations they found in their paper are important, these derivations are only valid for the sensing fields with symmetrical shapes, and furthermore, it is assumed that the $\mathrm{BS}$ is also symmetrically positioned relative to these symmetrically shaped sensing fields. However, in that study, we observe that these special cases lack the real practice experience. For example, the case that gives $E\left[d_{t o B S}\right]$, when the sensing field is any non-regular polygon and/or when the BS is located arbitrarily outside the field, is not covered by their formulations. Thus, a general-purpose derivation reflecting many real-life scenarios is missing and therefore there is a gap to be filled.

Herein, one of our contributions is to close this gap by deriving $E\left[d_{t o B S}\right]$ for the above mentioned special case. It is worth mentioning that the value of $E\left[d_{t o B S}\right]$ simply represents the mean (hereinafter, the terms, mean and average, will be used interchangeably in the remainder of the paper) distance $\left(\mu_{d}\right)$ of the whole population (i.e., all points 
in the sensing field). However, instead of finding out the mean distance of the whole population, it will be more practical to derive the mean distance of a certain sample size, specifically for random deployment scenarios. For this reason, we also extend the preliminary analysis in our previous work in Sevgi and Ali (2014) to enable a network designer to estimate mean distance $(\bar{d})$ for a sample size of $N$. The sample here indicates one realization of a deployment with $N$ sensor nodes.

Another motivation of this study is that our $E\left[d_{t o B S}\right]$ and $\bar{d}$ derivations serve as useful tools to reduce the overhead in the existing techniques and simulations that exclusively aim to estimate these values as part of their algorithms. The findings in this paper can be used in existing cluster-based architectures Gong et al. (2013), Chen et al. (2014), and Sun et al. (2011) either to calculate or to estimate the average distance between sensor nodes and the BS as a part of their clustering algorithm. Our findings might reduce the overhead in the existing techniques and simulations that exclusively aim to estimate $\bar{d}$ or calculate $E\left[d_{t o B S}\right]$.

\section{System Model}

In this section, the general system model is provided, and the relevant assumptions and the preliminaries used throughout the paper to facilitate the derivation of $E\left[d_{t o B S}\right]$ and $\bar{d}$ expressions are given.

\subsection{Network Model and Assumptions}

In this study, we assume a model suited for a stationary WSN application where $N$ sensor nodes are deployed randomly and uniformly over a sensing field. We consider that the shape of the sensing field can be any simple $n$-sided polygon. The main reason behind this assumption is that a given simple polygon has at least one triangulation. We discuss diagonalization and triangulation of a simple polygon in detail later in the subsequent subsection. Considering this attribute of a simple polygon, the analytical findings derived in this paper can easily be generalized for the most of the WSN deployment scenarios that employ numerous different shapes. For example, when $n$ is 4 in an $n$-sided polygon, the shape of sensing field may be a square, a rectangle, a trapezoid, or any other quadrilateral. Apart from these wellknown polygon shapes, the sensing field may also be any arbitrary polygon. For instance, if $n$ is 9 in an $n$-sided polygon, then the shape of the sensing field is a nonagon as shown in Figure 1. Therefore, having this flexibility of choosing the shape of the sensing field from many different shapes can reflect real-life scenarios in a better way. This general-purpose assumption is one key contribution of this study.

Recall that, in a typical WSN, the sensor nodes are designed to monitor the sensing field and to forward the sensed data to the BS. Therefore, the location of the BS, the orientation of the BS relative to the sensing field, the number of the BSs deployed, and the mobility of the BS and the nodes are of crucial importance. As such, in our network model, it is assumed that there is only one $B S$ whose location is fixed (i.e., stationary). Similar to the BS, each sensor node is assumed to be stationary and, additionally, they are unattended. Moreover, we consider that the BS is positioned arbitrarily outside or inside the sensing field.
In an illustrative example, the BS may be located outside the sensing field and near one of the edges as it can be seen in Figure 1 (a) and (c), or it may be positioned to a location again outside the field but near the middle as shown in Figure 1 (b). At this point, it is also worth mentioning that each Point $O$ shown in Figure 1 is the coordinate of the BS and at the same time it is assumed to be the origin of any coordinate system relative to the orientation of the sensing field. In fact, the origin point according to which a BS is assumed to be located is not specifically important. It is solely a reference point for the coordinate system used in the calculations. Therefore, we believe that our assumption on relative orientation of a BS can be used frequently in real-life deployment scenarios.

As for the last assumption, let coordinates of BS and each vertex of the polygonal sensing field be known in advance before the deployment. Here, knowing the coordinates of the vertexes is essential since these values are required for the derivation of $E\left[d_{t o B S}\right]$ after triangulation of the polygon.

\subsection{Preliminaries}

In this subsection, we provide the notations used in Table 1. In order to be consistent with the related literature, we rely on the notations used in Amini et al. (2012). The reader should note that we use three different expectation value functions: $E\left[d_{t o B S}\right], E\left[d_{P t o B S}\right]$, and $E\left[d_{T_{i} \text { toBS }}\right]$. The letter $E$ stands for the expected value function for the variable in square brackets. The letter $d$ in the square brackets specifies the distance function, while the letters $\left(P\right.$ and $\left.T_{i}\right)$ on the right of the letter $d$ represent the sensing field under study. The remaining part, "toBS", indicates the base station whose coordinates will be used to calculate the distance between itself and each point in the sensing field. If no letter is used on the left of the letter $d$, this means the entire sensing field is considered.

Table 1 Summary of notation

\begin{tabular}{|l|l|}
\hline Symbol & Definition \\
\hline$N$ & $\begin{array}{l}\text { The number of sensor nodes deployed } \\
\text { randomly over a given sensing field }\end{array}$ \\
\hline$P$ & $\begin{array}{l}\text { A simple } n \text {-sided polygon shaped sensing } \\
\text { field }\end{array}$ \\
\hline$T_{i}$ & The $i^{\text {th }}$ triangle after the triangulation of $P$ \\
\hline$A$ & $\begin{array}{l}\text { The surface area of the polygon shaped } \\
\text { sensing field } P\end{array}$ \\
\hline$A_{i}$ & The surface area of the $i^{\text {th }}$ triangle \\
\hline$E\left[d_{P t o B S}\right]$ & $\begin{array}{l}\text { The expected distance between each point } \\
\text { within } P \text { and the BS }\end{array}$ \\
\hline$E\left[d_{T_{i} t o B S}\right]$ & $\begin{array}{l}\text { The expected distance between each point } \\
\text { within } i^{t h} \text { triangle, } T_{i} \text {, and the BS }\end{array}$ \\
\hline $\bar{d}$ & $\begin{array}{l}\text { The mean distance between } N \text { randomly } \\
\text { deployed sensor nodes over a given sensing } \\
\text { field }\end{array}$ \\
\hline
\end{tabular}

When we use $E\left[d_{t o B S}\right]$, we mean the average distance between each point in the sensing field and the BS. On the other hand, when we use $\bar{d}$, we express the average distance between the given number of sensor nodes in the sensing field and the BS. 


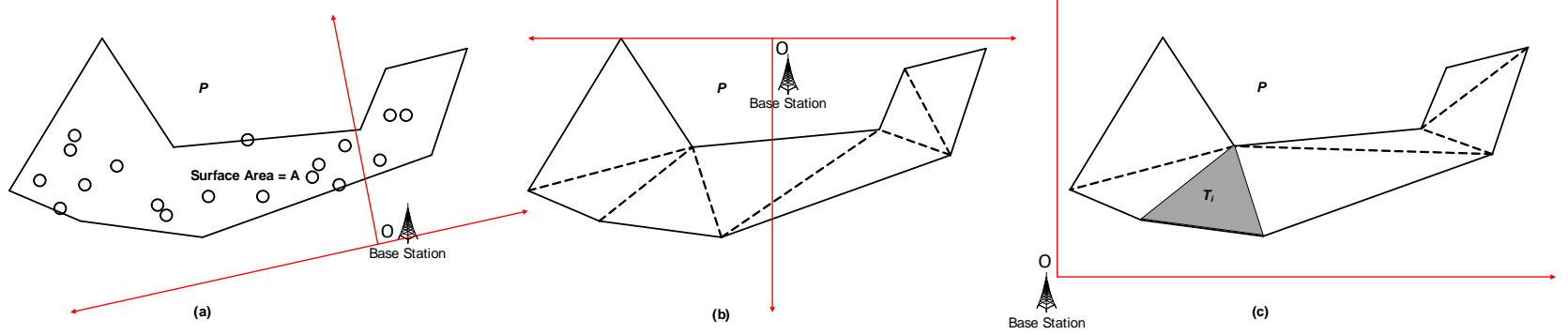

Figure 1 (a) Randomly deployed nodes over a simple 9-sided polygon sensing field $P$ (b) One possible triangulation of $P$ (c) Another triangulation of $P$

\subsection{Triangulation of a Simple Polygon}

Recall that our objective is to derive $E\left[d_{t o B S}\right]$ formulation for a given polygon shaped sensing field. Thus, let sensing field $P$ be a simple $n$-sided polygon in the plane, not necessarily a convex shape (Garey et al. (1978)). One of the most important features of a simple polygon is that it has a welldefined bounded interior and an unbounded exterior, where the interior is surrounded by edges as stated in Weisstein, E. (2016). Note also that a diagonal of $P$ is a line segment joining two non-adjacent vertices of $P$. The triangulation of the polygon is defined in (de Berg et al. (2008)) as the decomposition of a polygon into triangles by a maximal set of non-intersecting diagonals. The authors also showed the proof of a related theorem in which every simple polygon admits a triangulation, and any triangulation of a simple polygon with $n$ vertices consists of exactly $n-2$ triangles. This proof actually implies that a simple polygon must have at least one triangulation. Often a simple polygon has more than one triangulation. Multiple triangulations for a polygon can be exemplified by the two triangulations as illustrated in Figure 1 (b) and (c).

The reflection of this proof to the WSN domain regarding the existence of triangulation of a simple polygon is significant. This is because triangulation facilitates the analysis and the estimation of system parameters in WSNs. These system parameters are often functions of the polygon shaped sensing field under study. For example, suppose that we have a sensing field with a fairly complex shape similar to the 9-sided polygon given in Figure 1 and an arbitrarily positioned BS. First, we have to triangulate the 9-sided polygon. For each triangle, we should calculate the $E\left[d_{T_{i} t o B S}\right]$ which will be explained in the next section. If one encounters a difficulty in solving the integral in $E\left[d_{T_{i} t o B S}\right]$, the triangles can be divided into further triangles to make it easier to integrate. Once all the integrations for each triangle are calculated, their average values can be computed to reach $E\left[d_{\text {toBs }}\right]$ for the entire sensing field that has been initially triangulated.

\section{Expected Distance between each point in a Polygon and the BS $-E\left[d_{P t o B S}\right]$}

To find the expected distance, $E\left[d_{P t o B S}\right]$, between each point within polygon shaped (the terms, polygon and simple polygon, will be used interchangeably in the remainder of the paper) sensing field, $P$, and the arbitrarily located BS, one should integrate the product of two functions over $P$ in the Cartesian Coordinates as shown in the Eqn. 1.

$$
E\left[d_{P t o B S}\right]=\iint p(x, y) \cdot f(x, y) \cdot d x \cdot d y
$$

However, it is not easy to formulate such a generalized integration over a given polygon because the shape of each polygon depends on the measures of the interior angles between adjacent sides and the number of the sides it has. Thus, instead of using the given polygon in the integration directly, it is necessary to divide this polygon into smaller and integrable shapes. It is evident that a triangle is an atomic unit as is discussed in Section 3.2 and has an easyto-integrate shape in any given polygon shaped sensing field.

Considering the benefits of the triangulation and assuming that we have $k$ triangles after the triangulation of $P$, one should start with calculating $E\left[d_{T_{i} \text { toBS }}\right]$ from $1^{\text {st }}$ triangle till to $k^{\text {th }}$ triangle, and then find the average of $E\left[d_{T_{i} t o B S}\right]$ values for all triangles. This average value given in Eqn. 2 actually gives the $E\left[d_{P t o B S}\right]$ value for the entire polygon shaped sensing field.

$$
E\left[d_{P t o B S}\right]=\frac{1}{A} \sum_{i=1}^{k} E\left[d_{T_{i} t o B S}\right] A_{i}
$$

The reader should note that the value of $E\left[d_{T_{i} \text { toBS }}\right]$ given in Eqn. 2 is formulated in Eqn. 3.

$$
E\left[d_{T_{i} t o B S}\right]=\iint p(x, y) \cdot f(x, y) \cdot d x \cdot d y
$$

The first function in $E\left[d_{T_{i} t o B S}\right]$ in Eqn. 3 identifies the probability of a point being at a specific location within $i^{\text {th }}$ triangle, $T_{i}$. The second function determines the distance between the given point and the BS. First, we attempt to integrate the double integral in the Cartesian Coordinates as shown in Figure 2 (a). Note again that the relative location of the $\mathrm{BS}$ is assumed to be at the origin (Point $O$ in Figure 2 (a)) and it is known in advance before the deployment. Moreover, the coordinates of the vertex, $V_{l}, V_{2}$, and $V_{3}$, of this triangle illustrated in Figure 2 (a) are $\left(X_{1}, Y_{1}\right),\left(X_{2}, Y_{2}\right)$ and $\left(X_{3}, Y_{3}\right)$, respectively. At this point, it is important to state that the triangle analysed in Figure 2 can be thought as the shaded triangle obtained after the polygon triangulation which is illustrated in Figure 1 (c).

Let the infinitesimal rectangular segment in the Cartesian Coordinates be $d A=d x$. $d y$. This is a rectangle whose side lengths are $d x$ and $d y$. Suppose that the probability of a point being at location $(x, y)$, which is $\sqrt{x^{2}+y^{2}}$ units away from the BS, is $p(x, y)$. Since the probability of having that point at each location within the sensing field is identical, $p(x, y)$ is independent of $x$ and $y$ and is equal to $1 / A_{i}$. 


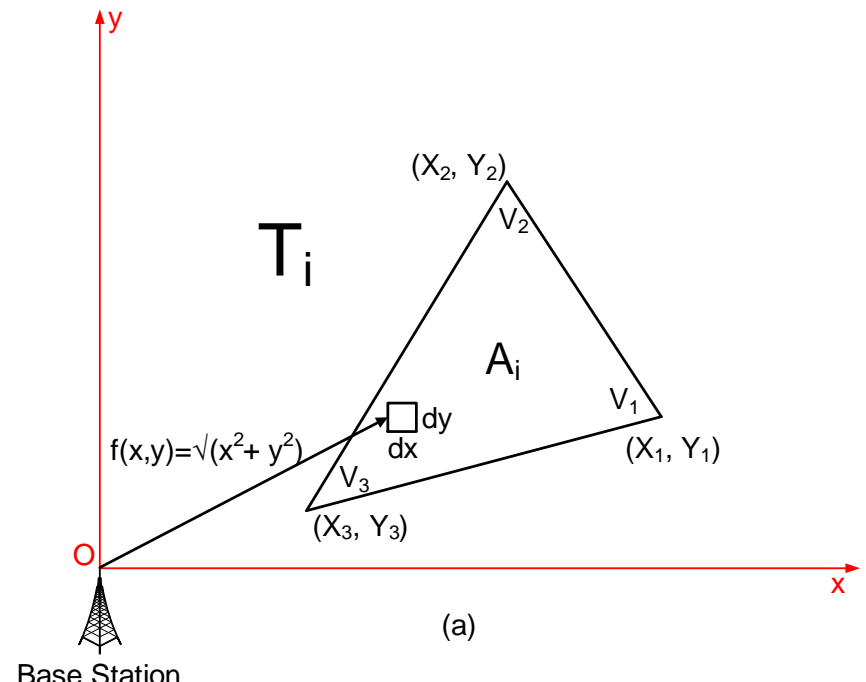

Base Station

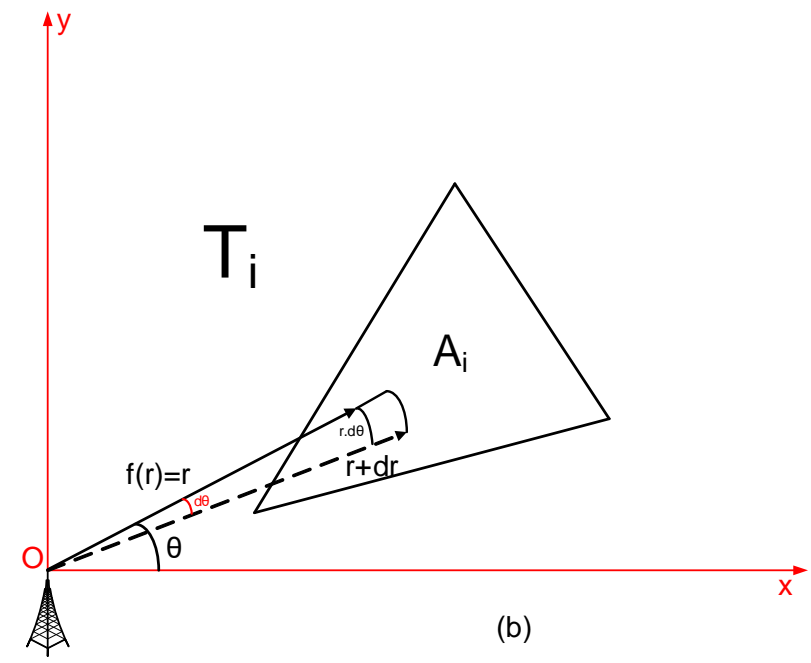

Base Station

Figure 2 Integration of $E\left[d_{T_{i} t o B S}\right]$ over a triangular field shown in the (a) Cartesian Coordinates (b) Polar Coordinates

This is because the nodes are randomly and uniformly deployed and the sum of these probabilities is 1 . However, any known (not necessarily uniform) probability distribution can be plugged into this function in the integration and the rest of the approach pursued in the paper will still be the same. By substituting $1 / A_{i}$ value with $p(x, y)$, the integration to find $E\left[d_{T_{i} t o B S}(x, y)\right]$ in the Cartesian Coordinates can be written as:

$$
\begin{aligned}
E\left[d_{T_{i} t o B S}(x, y)\right] & =\iint_{0} p(x, y) \sqrt{x^{2}+y^{2}} \cdot d x \cdot d y \\
& =\frac{1}{A_{i}} \iint \sqrt{x^{2}+y^{2}} \cdot d x \cdot d y
\end{aligned}
$$

However, it is not easy to integrate $E\left[d_{T_{i} t o B S}(x, y)\right]$ in Eqn. 4 using the Cartesian Coordinates. Then, we attempt to solve the same problem by using the Polar Coordinates and by integrating an infinitesimal ring shaped segment $d A=$

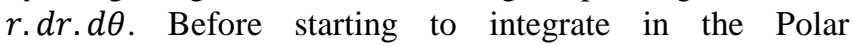
Coordinates, note again that this time the probability of a point node being in this ring shaped segment, which is $r$ radial distance from the BS, is $p(r)$ as is shown in Figure 2 (b). Owing to the same argument in the Cartesian Coordinates, the probability value $p(r)$ is also equal to $1 / A_{i}$. Therefore, we have:

$$
\begin{aligned}
E\left[d_{T_{i} t o B S}(r, \theta)\right] & =\iint_{0} p(r) \cdot r \cdot d r \cdot d \theta \\
& =\frac{1}{A_{i}} \iint r^{2} \cdot d r \cdot d \theta
\end{aligned}
$$

In what follows, we describe how to integrate $r^{2} \cdot d r . d \theta$ expression in Eqn. 5 over the $i^{\text {th }}$ triangle in detail.

\subsection{Derivation of $E\left[d_{T_{i} t o B S}\right]$ : Generic Case}

To facilitate our analysis, we introduce a static BS and a triangular sensing field, $T_{i}$, whose surface area is $A_{i}$ as shown in Figure 3. Moreover, the location of the BS is assumed to be located at the origin (Point $O$ in Figure 3). The coordinates of the vertexes, $V_{l}, V_{2}$, and $V_{3}$, of this triangle illustrated in Figure 3 are $\left(X_{1}, Y_{I}\right),\left(X_{2}, Y_{2}\right)$ and $\left(X_{3}, Y_{3}\right)$, respectively. It is also seen that the base side of triangle is tilted $\alpha$ degrees relative to the $\mathrm{x}$-axis and none of its sides is on the axis of symmetry. We call this deployment scenario as the generic case.

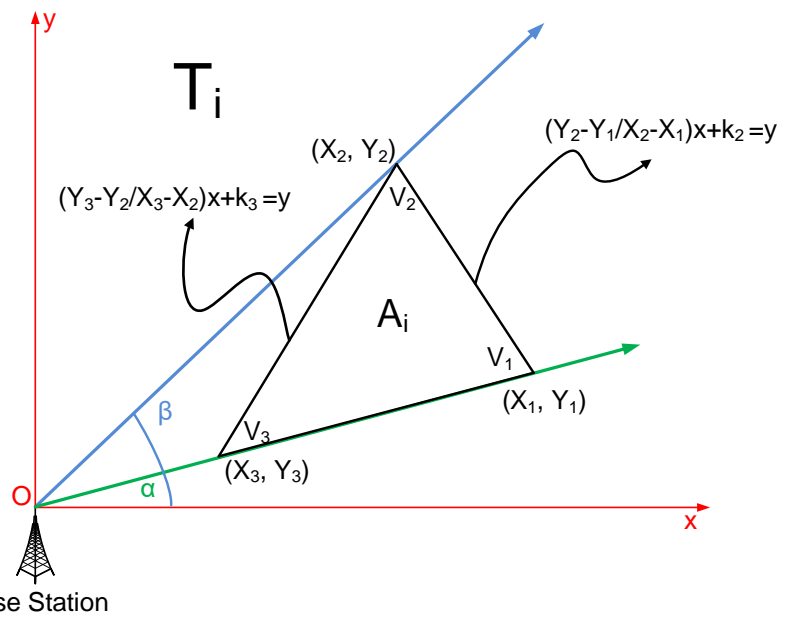

Figure 3 One orientation of the BS relative to the triangle $T_{i}$ (none of the sides is on the axis of symmetry) General Case

Herein, we start the derivation of $E\left[d_{T_{i} t o B S}\right]$ by mapping the limits of the definite integral in the Cartesian Coordinates into the limits in Polar Coordinates. (See Figure 3).

As far as the Polar Coordinates system is concerned, we need to find the limits of $r$ by holding $\theta$ fixed and let $r$ increase (since we are integrating with respect to $r$ ). As the point moves, it traces out a ray going out from the origin. Thus, the integration for $E\left[d_{T_{i} t o B S}\right]$ starts from $r_{l}$, where the ray enters region $A_{i}$, and ends with $r_{2}$, where the ray leaves this region. Since the ray enters $A_{i}$ at a point on $y=\frac{Y_{3}-Y_{2}}{X_{3}-X_{2}} x+$ $k_{3}$ line and exits $A_{i}$ at a point on $y=\frac{Y_{2}-Y_{1}}{X_{2}-X_{1}} x+k_{2}$ line in the Cartesian Coordinates, the trigonometric substitutions, $r_{1}=k_{3} /\left(\sin \theta-\frac{Y_{3}-Y_{2}}{X_{3}-X_{2}} \cos \theta\right) \quad$ and $\quad r_{2}=$ $k_{2} /\left(\sin \theta-\frac{Y_{2}-Y_{1}}{X_{2}-X_{1}} \cos \theta\right)$, are used to map these line 
equations to the Polar Coordinates. Here, the $k_{1}, k_{2}$, and $k_{3}$ are the y-intercepts of the three lines that form the triangle $T_{i}$.

Similarly, the lower and upper limits of $\theta$ can be written as $\alpha$ and $\beta$ respectively, as seen in Figure 3.

After plugging the limits of $r$ and $\theta$ into Eqn. 5, we have:

$$
E\left[d_{T_{i} \text { toBS }}\right]=\frac{1}{A_{i}} \int_{\alpha}^{\beta} \int_{\frac{k_{2}}{\left(\sin \theta-\frac{Y_{2}-Y_{1}}{X_{2}-X_{1}} \cos \theta\right)}}^{\frac{k_{3}}{\left(\sin \theta-\frac{Y_{3}-Y_{2}}{X_{3}-X_{2}} \cos \theta\right)}} r^{2} \cdot d r \cdot d \theta
$$

where area, $A_{i}$, is a constant and given in Eqn. 7 (Weisstein, E. (2017))

$$
\begin{aligned}
A_{i}=\frac{1}{2}\left(-X_{2} Y_{1}+\right. & X_{3} Y_{1}+X_{1} Y_{2}-X_{3} Y_{2}-X_{1} Y_{3} \\
& \left.+X_{2} Y_{3}\right)
\end{aligned}
$$

Substitute

$$
t_{3}=\frac{Y_{3}-Y_{2}}{X_{3}-X_{2}} \text { and } t_{2}=\frac{Y_{2}-Y_{1}}{X_{2}-X_{1}}
$$

Plug $t_{2}$ and $t_{3}$ into solved integrals:

$$
E\left[d_{T_{i} t o B S}\right]=\left.\frac{1}{3 \cdot A_{i}} \int_{\alpha}^{\beta} r^{3}\right|_{r=\frac{k_{3}}{\left(\sin \theta-t_{3} \cos \theta\right)}} ^{r=\frac{k_{2}}{\left(\sin \theta-t_{2} \cos \theta\right)}} d \theta
$$

Now solving

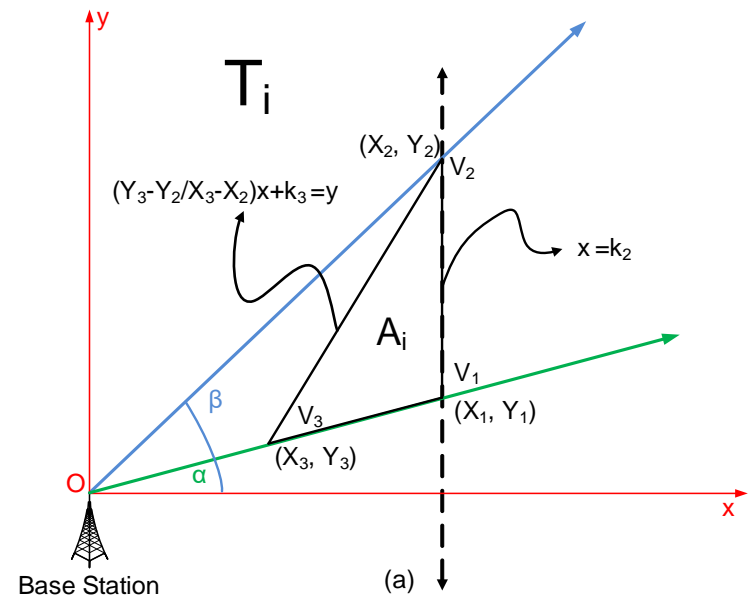

$$
\begin{aligned}
E\left[d_{T_{i} t o B S}\right]=\frac{1}{3 \cdot A_{i}} & {\left[k_{2}^{3} \int_{\alpha}^{\beta} \frac{d \theta}{\left(\sin \theta-t_{2} \cos \theta\right)^{3}}\right.} \\
& \left.-k_{3}^{3} \int_{\alpha}^{\beta} \frac{d \theta}{\left(\sin \theta-t_{3} \cos \theta\right)^{3}}\right]
\end{aligned}
$$

We introduce the following substitutions:

$$
\begin{aligned}
Z_{t_{2}}(\theta) & =\int \frac{d \theta}{\left(\sin \theta-t_{2} \cos \theta\right)^{3}} \\
Z_{t_{3}}(\theta) & =\int \frac{d \theta}{\left(\sin \theta-t_{3} \cos \theta\right)^{3}}
\end{aligned}
$$

Finally, we have the formulation for one of our research problems after substituting the upper and lower bounds for $\alpha$ and $\beta$, given in Eqn.12:

$$
\begin{array}{r}
E\left[d_{T_{i} t o B S}\right]=\frac{1}{3 \cdot A_{i}}\left[k_{2}^{3}\left(Z_{t_{2}}(\beta)-Z_{t_{2}}(\alpha)\right)\right. \\
\left.-k_{3}^{3}\left(Z_{t_{3}}(\beta)-Z_{t_{3}}(\alpha)\right)\right]
\end{array}
$$

Note that $Z_{t_{2}}(\theta)$ and $Z_{t_{3}}(\theta)$ in Eqn. 12 could not be replaced explicitly with their exact values. They are presented in Appendix A-1 due to the size limitation of this paper and the readability concerns.

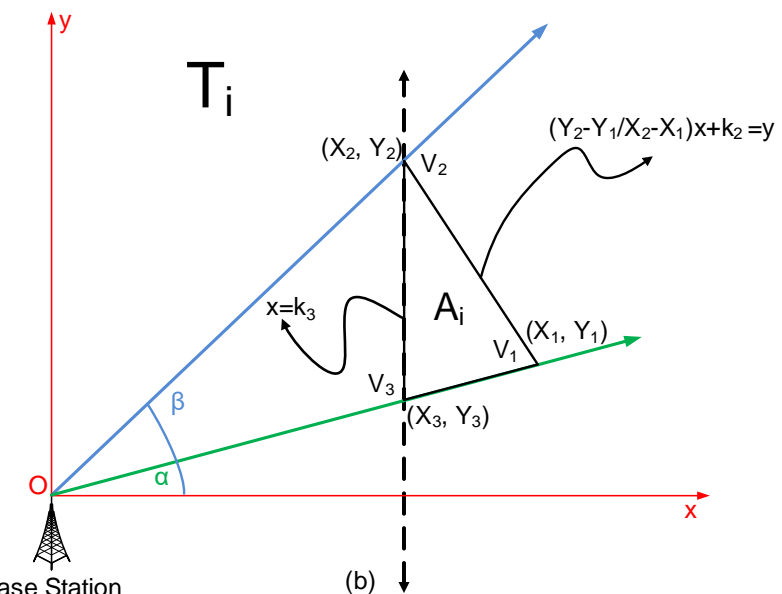

Figure 4 Two orientations of the BS relative to relative to a triangle $T_{i}$ (only one side is on the axis of symmetry) (a) Case 1-A

(b) Case 1-B
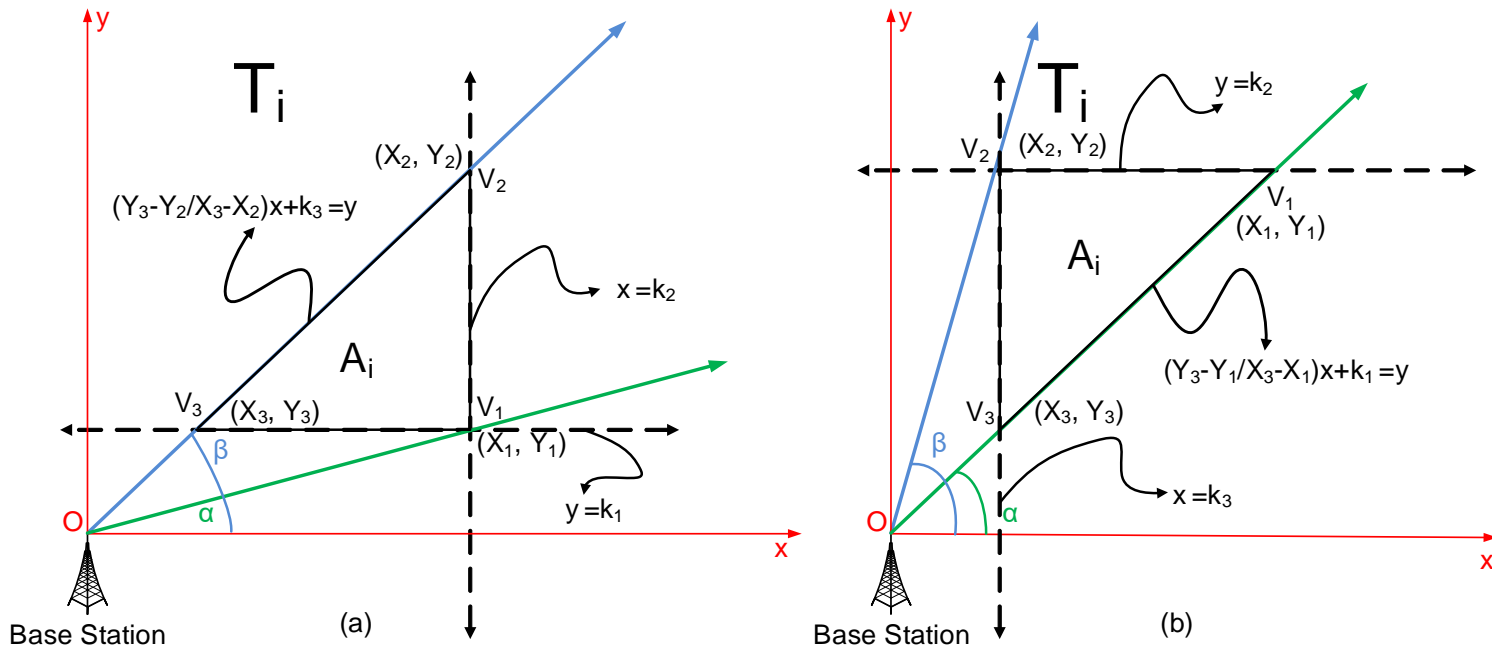

Figure 5 Two orientations of the BS relative to a right-angle triangle $T_{i}$ (two sides are on the axis of symmetry) (a) Case 2-A (b) Case 2-B 


\subsection{Derivation of $E\left[d_{T_{i} t o B S}\right]$ : Specific Cases}

In the previous section, a triangle is considered after the triangulation of a polygon shaped sensing field. The scenario given in Figure 1 describes a generic triangle none of whose sides are on the axis of symmetry. In other words, there is a certain degree of angle between the sides of the triangle and the axis. In this section, we consider the deployment scenarios where the $\operatorname{side}(s)$ of the triangle is/are either parallel to or on the axis.

Case 1: Assume that we have a triangular shaped sensing field whose only one side is on the axis of symmetry as is shown in Figure 4 (a) or (b). Then, we can find $E\left[d_{T_{i} t o B S}\right]$ value with the same approach described in the previous subsection.

Case 1-A: For a deployment scenario as illustrated in Figure 4 (a), the $E\left[d_{T_{i} t o B S}\right]$ value is formulated in Eqn. 13.

$$
\begin{aligned}
& E\left[d_{T_{i} t o B S}\right]=\frac{1}{A_{i}} \int_{\alpha}^{\beta} \int_{\frac{k_{2}}{\cos \theta}} r^{2} \cdot d r \cdot d \theta \\
& E\left[d_{T_{i} \text { toBS }}\right]=\frac{1}{3 \cdot A_{i}}\left[k_{2}^{3} \int_{\alpha}^{\beta} \frac{d \theta}{(\cos \theta)^{3}}\right. \\
& \left.-k_{3}^{3} \int_{\alpha}^{\beta} \frac{d \theta}{\left(\sin \theta-t_{3} \cos \theta\right)^{3}}\right] \\
& \int \frac{d \theta}{(\cos \theta)^{3}}=C(\theta) \\
& =\frac{\ln (|\tan (\theta)+\sec (\theta)|)+\sec (\theta) \tan (\theta)}{2}+C \\
& E\left[d_{T_{i} t o B S}\right]=\frac{1}{3 \cdot A_{i}}\left[k_{2}^{3}(C(\beta)-\mathrm{C}(\alpha))\right. \\
& \left.-k_{3}^{3}\left(Z_{t_{3}}(\beta)-Z_{t_{3}}(\alpha)\right)\right]
\end{aligned}
$$

where $C(\beta)$ and $\mathrm{C}(\alpha)$ can be found by substituting the upper and lower bounds for $\beta$ and $\alpha$ given in Eqn. 15 .

Case 1-B: For a very similar deployment scenario described in Figure 4 (a), consider that we have a triangular sensing field located as illustrated in Figure 4 (b). Then the $E\left[d_{T_{i} t o B S}\right]$ value is formulated in Eqn. 17 and 18.

$$
\begin{gathered}
E\left[d_{T_{i} t o B S}\right]=\frac{1}{A_{i}} \int_{\alpha}^{\beta} \int_{\frac{k_{3}}{\cos \theta}}^{\frac{k_{2}}{\left(\sin \theta-\frac{Y_{2}-Y_{1}}{X_{2}-X_{1}} \cos \theta\right)}} r^{2} \cdot d r \cdot d \theta \\
E\left[d_{T_{i} t o B S}\right]=\frac{1}{3 \cdot A_{i}}\left[k_{2}^{3}\left(Z_{t_{2}}(\beta)-Z_{t_{2}}(\alpha)\right)\right. \\
\left.\quad-k_{3}^{3}(C(\beta)-\mathrm{C}(\alpha))\right]
\end{gathered}
$$

Case 2: In this particular deployment scenario, assume that we have a triangular shaped sensing field whose two sides are on the axis of symmetry as is shown in Figure 5 (a) or (b). Then, we can find $E\left[d_{T_{i} t o B S}\right]$ value with the same approach described in the previous subsection.

Case 2-A: For a deployment scenario as illustrated in Figure 5 (a), the $E\left[d_{T_{i} t o B S}\right]$ value is formulated in Eqn. 19.

$$
\begin{gathered}
E\left[d_{T_{i} \text { toBS }}\right]=\frac{1}{A_{i}} \int_{\alpha}^{\beta} \int_{\frac{k_{1}}{\sin \theta}}^{\frac{k_{2}}{\cos \theta}} r^{2} \cdot d r \cdot d \theta \\
E\left[d_{T_{i} t o B S}\right]=\frac{1}{3 \cdot A_{i}}\left[k_{2}^{3} \int_{\alpha}^{\beta} \frac{d \theta}{(\cos \theta)^{3}}\right. \\
\left.\int \frac{d \theta}{(\sin \theta)^{3}}=S(\theta) \quad-k_{1}^{3} \int_{\alpha} \frac{d \theta}{(\sin \theta)^{3}}\right] \\
=-\frac{\ln (|\csc (\theta)+\cot (\theta)|)+\cot (\theta) \csc (\theta)}{2}+C \\
\frac{E\left[d_{T_{i} t o B S}\right]=\frac{1}{3 \cdot A_{i}}\left[k_{2}^{3}(C(\beta)-C(\alpha))\right.}{\left.-k_{1}^{3}(S(\beta)-S(\alpha))\right]}
\end{gathered}
$$

where $S(\beta)$ and $\mathrm{S}(\alpha)$ can be found by substituting the upper and lower bounds for $\beta$ and $\alpha$ given in Eqn. 21 .

Case 2-B: For a very similar deployment scenario described in Figure 5 (a), assume that we have a triangular sensing field located as illustrated in Figure 5 (b). Then the $E\left[d_{T_{i} t o B S}\right]$ value is formulated in Eqn. 17 and 18.

$$
\begin{array}{r}
E\left[d_{T_{i} \text { toBS }}\right]=\frac{1}{A_{i}} \int_{\alpha}^{\beta} \int_{\frac{k_{3}}{\cos \theta}}^{\frac{k_{2}}{\sin \theta}} r^{2} \cdot d r \cdot d \theta \\
E\left[d_{T_{i} t o B S}\right]=\frac{1}{3 \cdot A_{i}}\left[k_{2}^{3}(S(\beta)-S(\alpha))\right. \\
\left.\quad-k_{3}^{3}(C(\beta)-C(\alpha))\right]
\end{array}
$$

Through the derivations in section 4.1 for a generic case and derivations in section 4.2 for specific cases, we have covered $E\left[d_{T_{i} t o B S}\right]$ derivations for all the deployment scenarios. This generalized approach forms the basis of our a generalpurpose mathematical framework to find the expected distance value

\subsection{Derivation of Mean Distance $(\bar{d})$ for $N$ Randomly Deployed Sensor Nodes}

One can easily observe that derived $E\left[d_{T_{i} t o B S}\right]$ formulation is actually the mean distance $\left(\mu_{d}\right)$ between every point (the entire population) within the sensing field and an arbitrarily located BS. Moreover, $\mu_{d}$ is finite as the expectation is integrated over the finite domain, $A_{i}$. However, due to a cost constraint, it is not feasible to deploy a node on every point in a sensing field. Having considered this real-life constraint, let us further assume that $N$ sensor nodes are to be deployed randomly and uniformly over this finite field. In this case, the observed mean distance, which is the sample mean (i.e., sample size $=N$ ), is denoted as $\bar{d}$. Having the knowledge of $\bar{d}$ might be critical for a number of RDWSN applications because in that case a network designer has the opportunity to estimate the mean distance between these sensor nodes to the BS $(\bar{d})$ prior to the actual deployment. This is why our analytical derivation of $\bar{d}$ is another key contribution of this study. The remainder of this subsection will discuss how $\bar{d}$ is derived. 


\section{Average Distance Estimation in RDWSNs: An Analytical Study}

Owing to the central limit theorem, the sampling distribution of $\bar{d}$ will be normal or nearly normal with mean $\mu_{\bar{d}}$ and the standard deviation $\sigma_{\bar{d}}$, if the sample size is sufficiently large $(N>30)$. As far as the WSN applications are concerned, $N>30$ assumption makes sense. This is because the number of deployed nodes in a WSN application is typically ranging hundreds to thousands. Thus, it can safely be assumed that the sampling distribution of $\bar{d}$ is distributed normally $\left(N \sim\left[\mu_{\bar{d}}, \sigma_{\bar{d}}\right]\right)$ with mean $\mu_{\bar{d}}$ and the standard deviation $\sigma_{\bar{d}}$ which are shown below:

$$
\mu_{\bar{d}}=\mu_{d} \quad \text { and } \quad \sigma_{\bar{d}}=\frac{\sigma}{\sqrt{N}}
$$

$\mu_{d}$, which is actually equal to $E\left[d_{T_{i} \text { toBS }}\right]$, is already derived in Eqn. 12. Therefore, in order for our derivations to be used in practical cases, it is required that the population standard deviation $(\sigma)$ be specified. And, $\sigma$ can be calculated in terms of expected values as are given in Weisstein (2015).

$$
\sigma=\sqrt{E\left[d^{2}{ }_{T_{i} t o B S}\right]-E\left[d_{T_{i} t o B S}\right]^{2}}
$$

To find $\sigma$, one needs to proceed by finding out $E\left[d^{2}{ }_{T_{i} t o B S}\right]$ value, which is the solution of the integral in the Polar Coordinates as is given in Eqn. 27:

$$
\begin{aligned}
& E\left[d^{2}{ }_{T_{i} \text { toBS }}\right] \\
& =\frac{1}{A_{i}} \int_{\alpha}^{\beta} \frac{k_{2}}{\int_{\left(\sin \theta-\frac{Y_{2}-Y_{1}}{X_{2}-X_{1}} \cos \theta\right)}} r^{2} \cdot r \cdot d r \cdot d \theta \\
& \left(\sin \theta-\frac{Y_{3}-Y_{2}}{X_{3}-X_{2}} \cos \theta\right)
\end{aligned}
$$

Plug $t_{2}$ and $t_{3}$ (See Eqn. 8) into solved integrals:

$$
\begin{aligned}
& E\left[d^{2}{ }_{T_{i} \text { toBS }}\right]=\left.\frac{1}{4 \cdot A_{i}} \int_{\alpha}^{\beta} r^{4}\right|_{r=\frac{k_{3}}{\left(\sin \theta-t_{3} \cos \theta\right)}} ^{r=\frac{k_{2}}{\left(\sin \theta-t_{2} \cos \theta\right)}} d \theta \\
& E\left[d^{2}{ }_{T_{i} t o B S}\right]= \frac{1}{4 \cdot A_{i}}\left[k_{2}^{4} \int_{\alpha}^{\beta} \frac{d \theta}{\left(\sin \theta-t_{2} \cos \theta\right)^{4}}\right. \\
&\left.-k_{3}^{4} \int_{\alpha}^{\beta} \frac{d \theta}{\left(\sin \theta-t_{3} \cos \theta\right)^{4}}\right]
\end{aligned}
$$

We introduce the following substitutions:

$$
\begin{gathered}
B_{t_{2}}(\theta)=\int \frac{d \theta}{\left(\sin \theta-t_{2} \cos \theta\right)^{4}} \text { and } B_{t_{3}}(\theta) \\
=\int \frac{d \theta}{\left(\sin \theta-t_{3} \cos \theta\right)^{4}}
\end{gathered}
$$

After substituting the upper and lower bounds for $\alpha$ and $\beta$, we have Eqn. 19:

$$
\begin{aligned}
E\left[d^{2}{ }_{T_{i} t o B S}\right]= & \frac{1}{4 . A_{i}}\left[k_{2}^{4}\left(B_{t_{2}}(\beta)-B_{t_{2}}(\alpha)\right)\right. \\
& \left.-k_{3}^{4}\left(B_{t_{3}}(\beta)-B_{t_{3}}(\alpha)\right)\right]
\end{aligned}
$$

Note again that $B_{t_{2}}(\theta)$ and $B_{t_{3}}(\theta)$ in Eqn. 31 could not be replaced explicitly with their exact values. They are presented in Appendix (Section B) due to the size limitation of this paper and the readability concerns.

Finally, to find $\sigma$, one can easily plug $E\left[d^{2}{ }_{T_{i}}\right.$ toBS $]$ value given in Eqn. 29 and $E\left[d_{T_{i} \text { toBs }}\right]\left(\mu_{d}\right)$ value given in Eqn. 12 into Eqn. 26.

Since the sampling distribution of $\bar{d}$ is assumed to be normal with $N \sim\left[\mu_{\bar{d}}, \sigma_{\bar{d}}\right]$, the z-scores can be used for the estimation of $\bar{d}$. At this stage, it should be emphasized that our contribution is the estimation of the mean distance between a given number of nodes and the BS by using Eqn. 32 to the related literature.

$$
\begin{aligned}
& \bar{d} \in\left(\mu_{d} \pm \frac{\sigma}{\sqrt{N}}\right) \text { for } \% 68.27 \text { of the time } \\
& \bar{d} \in\left(\mu_{d} \pm \frac{2 \sigma}{\sqrt{N}}\right) \text { for } \% 95.45 \text { of the time } \\
& \bar{d} \in\left(\mu_{d} \pm \frac{3 \sigma}{\sqrt{N}}\right) \text { for } \% 99.73 \text { of the time }
\end{aligned}
$$

For the deployment scenarios given in Figure 4 and Figure $5, E\left[d^{2}{ }_{T_{i}}\right.$ toBS $]$ derivations can easily be found by the approach described in this section.

\section{Conclusion}

This paper proposes a general-purpose mathematical framework to find the expected distance value $\left(E\left[d_{\text {toBs }}\right]\right)$ between every point within any $n$-sided simple polygon shaped sensing field and an arbitrarily placed BS whose location is known in advance. The novelty of our work is that the sensing field under study does not need to have a wellknown shape (i.e., square, rectangle, trapezoid, etc.). Moreover, we do not enforce to locate the BS in the center on the perimeter or on the axis symmetry of the sensing field. That is, the location of the BS can be at any point inside or outside this field. Based on this generic scenario, we further provide a derivation for the estimation of mean distance (i.e., $\bar{d}$ ) for a given number of sensor nodes when these nodes are deployed randomly and uniformly. Aside from the pure theoretical interest, the derivation of $\bar{d}$ is important because estimation of $\bar{d}$ can be facilitated by network designers in real-life applications before the actual deployment. $E\left[d_{\text {toBs }}\right]$ and or $\bar{d}$ values are also required not only for the calculation of the optimum number of clusters in clustered RDWSNs, but also for the decision about whether a multi-hop or a direct communication should be devised or not. Moreover, the analytical derivation of $E\left[d_{t o B S}\right]$ value might reduce the overhead in the existing techniques and simulations that aim to estimate this value as part of their algorithms.

Last but not least, the use of our derivation is not limited to the WSN domain. It can also be used in any domain when there is a need for a probabilistic approach to find the average distance between any given number of points which are all assumed to be randomly and uniformly located in any $n$-sided simple polygon shaped region and at any point near this region.

As future research directions, we envision that the use of multiple BSs will be a promising direction for research. Based on the findings of this study, we intend to exploit our $E\left[d_{t o B S}\right]$ and $\bar{d}$ formulations to solve the following two research problems:

- How can our $E\left[d_{t o B S}\right]$ and $\bar{d}$ formulations be exploited during the selection process of clustering schemes and/or routing techniques, when the number of BSs is more than one?

- How can our $E\left[d_{t o B S}\right]$ and $\bar{d}$ formulations be exploited in the relocation problem for a given number of mobile $\mathrm{BS}(\mathrm{s})$ that maximize the network lifetime and/or maximize the throughput? 


\section{A Appendix}

\section{A-1 Derivation of $Z_{t_{2}}(\theta)$ and $Z_{t_{3}}(\theta)$}

In this section, we discuss a specific form of integral aforementioned in Section 4.1. For the sake of readability, $E\left[d_{T_{i} t o B S}\right]$ formulation in Eqn. 10 includes a substitution (i.e., $Z_{t_{n}}(\theta)$ ) to denote this specific form. The substitutions $Z_{t_{2}}(\theta)$ and $Z_{t_{3}}(\theta)$ are used to represent $\int \frac{d \theta}{\left(\sin \theta-t_{2} \cos \theta\right)^{3}}$ and $\int \frac{d \theta}{\left(\sin \theta-t_{3} \cos \theta\right)^{3}}$, which is the special case of $\int \frac{\mathrm{d} \theta}{(\sin (\theta)-a \cos (\theta))^{3}}$ when $a=t_{2}$ and $a=t_{3}$ respectively.

$$
Z_{a}(\theta)=\int \frac{d \theta}{(\sin (\theta)-a \cos (\theta))^{3}}
$$

By using the tangent half-angle substitution, we have:

$$
Z_{a}(\theta)=\int \frac{d \theta}{\left(\frac{2 \tan \left(\frac{\theta}{2}\right)}{\tan ^{2}\left(\frac{\theta}{2}\right)+1}-\frac{a\left(1-\tan ^{2}\left(\frac{\theta}{2}\right)\right)}{\tan ^{2}\left(\frac{\theta}{2}\right)+1}\right)^{3}}
$$

We can use integration by parts and substitute $u=$ $\tan \left(\frac{\theta}{2}\right) \rightarrow \mathrm{d} \theta=\frac{2}{\sec ^{2}\left(\frac{\theta}{2}\right)} \mathrm{d} u \quad d \theta=\frac{2}{u^{2}+1} \mathrm{~d} u$

$$
Z_{a}(u)=2 \int \frac{\left(u^{2}+1\right)^{2}}{\left(a u^{2}+2 u-a\right)^{3}} d u
$$

By Ostrogradsky's method, we have the following:

$$
\begin{aligned}
& Z_{a}(u) \\
& =\frac{1}{2\left(a^{2}+1\right)} \int \frac{d u}{a u^{2}+2 u-a} \\
& +\frac{\left(-a^{3}-2 a\right) u^{3}+\left(a^{2}-2\right) u^{2}+\left(2 a-a^{3}\right) u-a^{2}}{2 a^{2}\left(a^{2}+1\right)\left(a u^{2}+2 u-a\right)^{2}}
\end{aligned}
$$

$$
\begin{aligned}
& \int \frac{d u}{a u^{2}+2 u-a} \\
& =\int-\frac{a \mathrm{~d} u}{\left(-a u+\sqrt{a^{2}+1}-1\right)\left(a u+\sqrt{a^{2}+1}+1\right)}
\end{aligned}
$$

After factoring the denominator and performing partial fraction decomposition:

$$
\begin{aligned}
& =\int\left(\frac{a}{2 \sqrt{a^{2}+1}\left(a u-\sqrt{a^{2}+1}+1\right)}\right) \mathrm{d} u \\
& -\frac{a}{2 \sqrt{a^{2}+1}\left(a u+\sqrt{a^{2}+1}+1\right)} \mathrm{d}
\end{aligned}
$$

Then, linearity is applied:

$$
\begin{aligned}
& =\frac{a}{2 \sqrt{a^{2}+1}} \int \frac{d u}{a u-\sqrt{a^{2}+1}+1} \\
& -\frac{a}{2 \sqrt{a^{2}+1}} \int \frac{d u}{a u+\sqrt{a^{2}+1}+1}
\end{aligned}
$$

Finally, using integration by parts again and undoing the substitution $u=\tan \left(\frac{\theta}{2}\right)$, one can find out Eqn. 40 .

Having discussed the derivation of $Z_{a}(\theta)$, it is time to plug our $\tan \left(\frac{\beta}{2}\right)$ and $\tan \left(\frac{\alpha}{2}\right)$ values in Eqn. 40, when $\mathrm{a}=t_{2}$ and $\mathrm{a}=t_{3}$. The trigonometric identities of these $\theta$ values in terms of the coordinates of the vertexes of the triangle under study are given in Eqn. 41.

Furthermore, these identities are also plugged into $Z_{t_{2}}(\theta)$ and $Z_{t_{3}}(\theta)$ formulations, which are the original contributions of this paper.

Now solving

$$
\begin{aligned}
& Z_{a}(\theta)=\int \frac{d \theta}{(\sin \theta-a \cos \theta)^{3}} \\
& =\frac{\left(-a^{3}-2 a\right) \tan ^{3}\left(\frac{\theta}{2}\right)+\left(a^{2}-2\right) \tan ^{2}\left(\frac{\theta}{2}\right)+\left(2 a-a^{3}\right) \tan \left(\frac{\theta}{2}\right)-a^{2}}{a^{2}\left(a^{2}+1\right)\left(a \tan ^{2}\left(\frac{\theta}{2}\right)+2 \tan \left(\frac{\theta}{2}\right)-a\right)^{2}} \\
& +\frac{\ln \left(\left|a \tan \left(\frac{\theta}{2}\right)-\sqrt{a^{2}+1}+1\right|\right)-\ln \left(\left|a \tan \left(\frac{\theta}{2}\right)+\sqrt{a^{2}+1}+1\right|\right)}{2\left(a^{2}+1\right)^{\frac{3}{2}}}+C \\
& \tan \left(\frac{\beta}{2}\right)=\tan \left(\frac{\tan ^{-1} \frac{Y_{2}}{X_{2}}}{2}\right) \tan \left(\frac{\alpha}{2}\right)=\tan \left(\frac{\tan ^{-1} \frac{Y_{1}}{X_{1}}}{2}\right)
\end{aligned}
$$

\section{A-2 Derivation of $B_{t_{2}}(\theta)$ and $B_{t_{3}}(\theta)$}

Herein, we discuss a specific form of integral aforementioned in Section 4.3. For the sake of readability, $E\left[d^{2}{ }_{T_{i} \text { toBS }}\right]$ formulation in Eqn. 31 includes a substitution (i.e., $B_{t_{n}}(\theta)$ ) to denote this specific form. The substitutions $B_{t_{2}}(\theta)$ and $B_{t_{3}}(\theta)$ are used to represent $\int \frac{\mathrm{d} \theta}{\left(\sin \theta-\mathrm{t}_{2} \cos \theta\right)^{4}}$ and $\int \frac{\mathrm{d} \theta}{\left(\sin \theta-\mathrm{t}_{3} \cos \theta\right)^{4}}$, which is the special case of $\int \frac{\mathrm{d} \theta}{(\sin (\theta)-a \cos (\theta))^{4}}$ when $a=t_{2}$ and $a=t_{3}$ respectively.

$$
B_{a}(\theta)=\int \frac{d \theta}{(\sin (\theta)-a \cos (\theta))^{4}}
$$

$$
\begin{aligned}
B_{a}(\theta)=\int \frac{\mathrm{d} \theta}{(\sin (\theta)-a \cos (\theta))^{4}} \\
=\int \sec ^{2}(\theta) \cdot \frac{\tan ^{2}(\theta)+1}{(\tan (\theta)-a)^{4}} \mathrm{~d} x
\end{aligned}
$$

Substitute $u=\tan (\theta) \rightarrow \mathrm{d} \theta=\frac{1}{\sec ^{2}(\theta)} \mathrm{d} u$

$$
B_{a}(u)=\int \frac{u^{2}+1}{(u-a)^{4}} \mathrm{~d} u
$$

Substitute $v=u-a \rightarrow \mathrm{d} u=\mathrm{d} v$

$$
=\int\left(\frac{1}{v^{2}}+\frac{2 a}{v^{3}}+\frac{a^{2}+1}{v^{4}}\right) \mathrm{d} v
$$

After appling linearity, the power rule, and undoing substitution $v=u-a$ : 


$$
B_{a}(u)=-\frac{1}{u-a}-\frac{a}{(u-a)^{2}}-\frac{a^{2}+1}{3(u-a)^{3}}
$$

Undo substitution $u=\tan (x)$

$$
\begin{gathered}
B_{a}(\theta)=-\frac{1}{\tan (\theta)-a}-\frac{a}{(\tan (\theta)-a)^{2}} \\
-\frac{a^{2}+1}{3(\tan (\theta)-a)^{3}}
\end{gathered}
$$

Rewrite/simplify:

$$
\begin{aligned}
& B_{a}(\theta)=\int \frac{\mathrm{d} \theta}{(\sin (\theta)-a \cos (\theta))^{4}} \\
= & -\frac{3 \tan ^{2}(\theta)-3 a \tan (\theta)+a^{2}+1}{3(\tan (\theta)-a)^{3}}+C
\end{aligned}
$$

Having discussed the derivation of $B_{a}(\theta)$, it is time to plug our $\tan \beta$ and $\tan \alpha$ values in Eqn. 31 when $\mathrm{a}=t_{2}$ and $\mathrm{a}=t_{3}$. The trigonometric identities of these in terms of the coordinates of the vertexes of the triangle under study are given below in Eqn. 42.

$$
\tan \beta=\frac{Y_{2}}{X_{2}} \quad \tan \alpha=\frac{Y_{1}}{X_{1}}
$$

\section{References}

Afsar, M. M. and Tayarani-Najaran, M.-H. (2014). Clustering in Sensor Networks: A literature survey. Journal of Network and Computer Applications, 46(0):198 - 226.

Akkaya, K., Younis, M., and Youssef, W. (2007). Positioning of base stations in Wireless Sensor Networks. IEEE Communications Magazine, 45(4):96-102.

Alia, O. M. (2017). Dynamic relocation of mobile base station in Wireless Sensor Networks using a cluster-based harmony search algorithm. Information Sciences, 385386:76-95.

Amini, N., Vahdatpour, A., Xu, W., Gerla, M., and Sarrafzadeh, M. (2012). Cluster size optimization in sensor networks with decentralized cluster-based protocols. Computer Communications, 35(2):207-220.

Amini, N., Vahdatpour, Alireza, Dabiri, Foad, Noshadi, Hyduke, and Sarrafzadeh, M. (2011). Joint consideration of energy-efficiency and coverage preservation in microsensor networks. Wireless Communications and Mobile Computing, 11(6):707-722.

Chen, H.-L., Chen, T.-A., and Hu, S.-H. (2014). A convergent algorithm for energy-balanced clusterheads selection in Wireless Sensor Networks. International Journal of Distributed Sensor Networks, 2014(719397):1-8.

de Berg, M., Cheong, O., van Kreveld, M., and Overmars, M. (2008). Computational Geometry Algorithms and Applications. Springer-Verlag Berlin Heidelberg, 3 edition.

Du, T., Qu, S., Liu, F., and Wang, Q. (2015). An energy efficiency semi-static routing algorithm for WSNs based on HAC clustering method. Information Fusion, 21:18-29.

Dwight, H. B. and Hedrick, E. R. (1956). Tables of Integrals and Other Mathematical Data. The Macmillan Company, 3 edition.
Garey, M. R., Johnson, D. S., Preparata, F. P., and Trajan, R. E. (1978). Triangulating a simple polygon. Information Processing Letters, 7(4):175-179.

Gong, B., Jiang, T., Xu, S., and Chen, P. (2013). An energyheterogeneous clustering scheme to avoid energy holes in Wireless Sensor Networks. International Journal of Distributed Sensor Networks, 2013(796549):1-8.

Guney, E., Aras, N., Altinel, I. K., and Ersoy, C. (2012). Efficient solution techniques for the integrated coverage, sink location and routing problem in Wireless Sensor Networks. Computers \& Operations Research, 39(7):1530-1539.

Heinzelman, W., Chandrakasan, A., and Balakrishnan, H. (2002). An application-specific protocol architecture for wireless microsensor networks. IEEE Transactions on Wireless Communications, 1(4):660-670.

Kulkarni, R. V. and Venayagamoorthy, G. K. (2011). Particle swarm optimization in Wireless-Sensor Networks: A brief survey. IEEE Transactions on Systems, Man, and Cybernetics, Part C (Applications and Reviews), 41(2):262267.

Maher, R., Murat, A. H., and Hichem, S. (2016). Exact methods for sensor deployment problem with connectivity constraint in Wireless Sensor Networks. International Journal of Sensor Networks, 21(3):157-168.

Qingguo, Z. and P., F. M. (2017). A two-phase coverageenhancing algorithm for hybrid Wireless Sensor Networks. Sensors, 17(117)

Sevgi, C. and Ali, S.A. (2014). On the Analysis of Expected Distance between Sensor Nodes and the Base Station in Randomly Deployed WSNs. In The Proceedings Internet and Distributed Computing Systems, 7th International Conference, IDCS 2014, pages 358-368.

Soroush, N., Hamidreza, G., Chee-Onn, C., and Hiroshi, I. (2012). A survey on the taxonomy of cluster-based routing protocols for homogeneous Wireless Sensor Networks. Sensors, 12(6):7350-7409.

Sun, Y., He, Y., Zhang, B., and Liu, X. (2011). An energy efficiency clustering routing protocol for WSNs in confined area. Mining Science and Technology (China), 21(6):845850 .

Tsai, Y. (2007). Coverage-preserving routing protocols for randomly distributed Wireless Sensor Networks. IEEE Transactions on Wireless Communications, 6(4):1240-1245.

Tyagi, S. and Kumar, N. (2013). A systematic review on clustering and routing techniques based upon LEACH protocol for Wireless Sensor Networks. Journal of Network and Computer Applications, 36(2):623 - 645.

Weisstein, E. (2015). Mathworld-a wolfram web resource, standard deviation.

http://mathworld.wol fram.com/StandardDeviation.html.

[Online; accessed 18-March-2018]. 
Sevgi

Weisstein, E. (2016). Mathworld-a wolfram web resource, Simple Polygon.

http://mathworld.wolfram.com/SimplePolygon.html [Online; accessed 18-March-2018].

Weisstein, E. (2017). Mathworld-a wolfram web resource, Triangle Area. http://mathworld.wolfram.com/TriangleArea.html [Online; accessed 18-March-2018].

Younis, M., Senturk, I. F., Akkaya, K., Lee, S., and Senel, F. (2014). Topology management techniques for tolerating node failures in Wireless Sensor Networks: A survey. Computer Networks, 58:254-283. 